\begin{abstract}
This article presents a review of over 100 studies relying on the Institutional Research Regime (IRR) framework. We base the review on a set of 13 elements that cover categories concerning research focus, research design and results. The article first presents the development of IRR studies over time by showing and discussing how different elements of the IRR framework are more or less popular in the literature, and how this popularity develops over time. For example, we have identified a trend in the IRR literature to focus more on actors and activities, while the resource focus predominates across time. Second, the article analyses how different elements of the IRR framework are combined in the existing literature and thus identifies three groups of IRR studies, that is, complex IRR studies, bottom-up IRR studies, and top-down IRR studies.
\end{abstract}

This document is the accepted manuscript version of the following article:

Lieberherr, E., Fischer, M., \& Tschannen, A. (2019). Taking stock of institutional resource regime research: a meta-analysis. Environmental science and Policy, 97, 81-89. https://doi.org/10.1016/j.envsci.2019.04.003 


\title{
Taking Stock of Institutional Resource Regime Research: A Meta- Analysis
}

\author{
Eva Lieberherra,*, Manuel Fischerb,c, Amadea Tschannena \\ a Institute for Environmental Decisions, Swiss Federal Institute of Technology (ETH), Universitaetstrasse 16, \\ 8092, Zurich, Switzerland \\ b Department of Environmental Social Sciences, Swiss Federal Institute of Aquatic Science and Technology \\ (Eawag), Überlandstrasse 133, 8600, Duebendorf, Switzerland \\ c Institute of Political Science, University of Berne, Fabrikstrasse 8, 3012, Berne, Switzerland
}

\section{Introduction}

The Institutional Resource Regime (IRR) framework (Knoepfel et al., 2001) has been applied in many different contexts since its inception in 1999. As any framework, the IRR consists of a set of different elements supposed to structure the analysis, in this case of natural resource governance. As such, researchers can - depending on the characteristics of the resource, the research question, and the case context - emphasize some elements of the framework over others, or even combine these elements in different ways (Schlager, 1999). What is more, we have observed an evolution over time in the focus that researchers put on different elements of the IRR framework: From an emphasis on historical legal screenings of different natural resources, such as water, forest, soil and air, applications of the framework have moved to an increasing focus on actors, their agency and action-resources. While remaining largely Europecentric, we also observe a growing application in countries of the global south in recent years. Furthermore, studies are extending the scope of resources analysed, methods applied to analyse the cases, and the theoretical assumptions and concepts used in conjunction with the IRR framework.

Against this background of the diversity of how researchers select elements of the IRR framework and the development of these choices over time, we propose to take stock of the existing IRR literature. To do so, we develop a catalogue of elements regarding the design, focus and results of IRR studies. More specifically, for over 100 publications, we code and analyse a set of variables assessing elements such as for example the importance of resources vs. actors, a top-down vs. bottom-up approach, or the relation of the IRR to sustainability. We code 13 elements as numerical categories. The analysis of the numerical variables relies on descriptive statistics to observe the distribution and development of single elements, as well as multiple correspondence analysis (MCA) that allows us to observe how different elements cluster together. 
This endeavour not only results in a first broad overview of IRR studies, but more importantly allows to identify similarities and differences across studies as well as patterns of combinations of empirical, theoretical, and methodological aspects. This in turn provides 1) an assessment of what we have learned thus far about natural resource governance through the IRR framework across the range of IRR applications, and 2) an input for identifying gaps in the existing research, developing questions for future research, and contributing to the development of the IRR framework.

The remainder of the article is structured as follows. We first present and discuss the catalogue of IRR elements that structure our review. We then provide information on the selection of publications included in the review and on the coding procedure. A first part of the results presents simple descriptive statistics on the distribution and development of the 13 elements over time. A second empirical part then analyses how different elements were combined in existing research. From there, we conclude with a discussion of key questions for future research and development of the framework.

\section{Catalogue of IRR elements}

The catalogue of elements is based on a screening of the key literature on the IRR (Aubin, 2007; 2008; de Buren, 2015; Dupuis and Knoepfel 2016; Gerber et al., 2009; Knoepfel et al., 2001; Nahrath and Brethaut 2016), as well as input from IRR scholars. This was an iterative process: we first screened the literature, starting with de Buren (2015), which provides an overview of IRR publications. We then contacted some of these key authors and obtained feedback on our preliminary elements as well as input regarding what further texts we could use to help develop and test our codebook. We then returned to the key literature mentioned above to finalize our catalogue of elements.

To structure the elements of the IRR that we code and analyse, we rely on three broad categories: research focus, research design, and results (see Appendix A for an overview of the elements). IRR elements related to the research focus are the following. First, we code whether an IRR application puts emphasis on a given resource and its use and services, or whether it addresses actors' activity that may affect different resources (1). In this sense IRR applications can be distinguished by whether the "entry point" to the analysis is the resource or the activity. That is, the research begins either by describing a resource such as soil, water, air or rather by addressing an activity such as wind power, nuclear waste management or road construction. Second, we look at the relevance of actors, that is, instead of focusing on (natural) 
resources, the actors - and more specifically their strategies and actor resources - are central in the analysis. (2). Third, we code relations at the actor level (3). Here we address use rivalries and/or conflicts, which may occur within one resource or between multiple resources. Fourth, we code whether a study investigates a natural resource such as water, soil or forest, or an artificial resource, such as collective housing or a railway (4). We also assess the main government level that influences the case (5), and we code whether an interplay between different government levels is addressed in the IRR study (6). Two further elements related to the research focus are the question of whether sustainability is mentioned or not related to the IR (7) and the kind of causal link that is made between the IR and sustainability (8): The theoretical literature describes two options regarding the direction of the causality between the institutional regime (IR) (for an explanation of IR, see Gerber et al., this issue) and sustainability (Gerber et al., this issue). The first (option 1) follows the assumption that the closer an IR moves towards an integrated regime (regulations and actors are aligned), the higher the probability is, that sustainable use conditions will be created and vice versa. The second (option 2) presupposes that the greater the threat to the stability of a resource, the more likely it will be perceived as a collective problem and the probability of making the regime more integrated increases.

Elements belonging to the research design are the following: First, we code whether the study considers the IR as the dependent or the independent variable, or both (9). As an additional element of research design, we assess whether an IRR study corresponds to a case study or a comparative study (10). We then analyse whether the time aspect is part of the research design (11), that is, whether a stable IR or a change of an IR are at the centre of the analysis. We further code whether the study relies on a top-down (starting with legal texts), on a bottom-up (starting with a resource and its use), or mixed perspective (12).

Finally, with respect to research results, we simply assess whether the general focus of the findings is on resources, actors, or both (13).

\section{Identification and coding of relevant research}

We coded extant IRR applications published between 2000 and 2017. We chose 2000 as our starting date as the first IRR publication was issued in this year. First, we conducted a Scopus, Web of Science and google scholar search with the term "institutional resource regime" and compared the findings with an overview publication of de Buren (2015). Our assessment of the literature includes published journal articles, as well as books and working papers published in 
institutional working paper series. ${ }^{1}$ Second, we did a snowball sampling with the identified publications (books, book chapters, working papers and articles) by systematically checking the reference lists of the publications in order to identify further relevant publications. We excluded publications that only refer to the framework in a footnote or as an argument in the theory section, but mainly apply other concepts and theories. ${ }^{2}$ An example is Nahrath (2000) who uses an approach very similar to the IRR but the analysis explicitly only relies on the "Common Pool Resource" (CPR) approach from Ostrom (1990). We further refrain from coding work that is purely theoretical or conceptual for lack of comparability with the empirical pieces of literature. An exception is the publication of Varone et al. (2002).We included this publication even though it has a theoretical focus, as it still provides empirical examples. The final sample comprises 110 publications.

In a pre-test, a selection of 10 publications differing in form, the analysed resource and language (English, German and French) were coded independently by the second and third authors of this article. We compared the coding results and adopted the code book, accordingly (e.g. made subcategories of our initial variables, included descriptive variables if an element was hard to code numerical). Because of the large share of French publications (37\%), we decided to involve a native speaker for the coding. Again, we did a pre-test with 5 publications to test the congruency of the coding of the third author of this article and the French speaking collaborator. To keep the coding constant, both coders met once a week to discuss any issues and to further adopt and specify the coding guidelines (e.g. make comments, add subcategories and illustrative examples).

Throughout the coding process, some variables remained difficult to code. For instance, many publications are inconsistent with the categorization of key terms such as artificial resource and sustainability or do not explicitly define the dependant/independent variables. In these cases, the piece of literature had to be coded based on implicit information according to the reading of the coder. To decrease the risk of errors and incongruent coding, these cases were systematically discussed between both researchers. The most challenging variable for coding was the type of resource under investigation and, more specifically, the category of artificial resources: First, these resources were sometimes labelled as artificial, as extended or

\footnotetext{
${ }^{1}$ Mainly the working papers published at IDHEAP (Institut de hautes études en administration publique) in Lausanne.

${ }^{2}$ The decision for in- or exclusion is based on screening of abstracts, keywords and refined search terms (e.g. regime, extent, coherence) if a pdf document was available. To further legitimate the decision of "exclusion" we checked the google scholar citation report of the publication.
} 
as secondary resources, and all include different natural (sometimes also called "basic") resources. Second, the definition of the scope of an artificial resource, that is, the goods and services relevant for the resource definition, and thus the policies included in a specific resource regime, differs between publications. This aspect makes it difficult to identify and compare coherence and extent of the resource regime(s), as these regime dimensions are directly related to the resource definition (for definitions of coherence and extent, see Gerber et al., this issue).

In our sample, we find a predominant focus on natural (70\%) rather than artificial resources. Among the natural resources, water (33\%), soil (23\%), forest (15\%) and landscape $(12 \%)$ are the most studied resources. In terms of applications to countries, the IRR framework has been mainly applied in French and German speaking countries, with more than half of the studies conducted in Switzerland (61\%), and further applications in Germany, France, Belgium, Spain, The Netherlands and Italy.

\section{Analysis}

We first conducted a descriptive analysis of the final sample (110 publications) using the 13 variables described above. The $\mathrm{N}$ varies across the elements analysed because not all publications provided relevant information for all elements. In addition, we add examples and specifications from some IRR publications to further illustrate our results based on the numerical variables. We do not present the descriptive results concerning these 13 variables in the order described above, but rather opt for a discussion of the variables according to similar developments over time or substantive relations between variables belonging to research focus, design or results.

\subsection{Partial shift toward activity and actors vs. resources}

The results on the research focus (resource vs. activity) indicate that a shift toward an "activity" approach occurred in 2007 (see figure 1). Up until 2007, the "resource" approach was dominant. All the articles had entered the field of analysis by looking at a specific (natural) resource, such as water, land, forest or air. In 2007, we see that scholars began to start with an activity (e.g. nuclear waste management or road construction) as an entry point to the analysis. Starting with an activity as the entry point does not exclude the resource(s), but rather helps to narrow the focus and defines the resource, while at the same time actors play a more important role. For instance, analysing nuclear waste management or road construction (Dupuis and Knoepfel, 2016, Savary, 2008) is much more specific than starting with soil and land. The latter (resource as the entry point) typically entails defining the resource, extensively cataloguing all 
products, making an inventory of all the uses, and so on (see de Buren, 2015). The activity approach, by contrast, starts with a rather specific definition of the resource, the products, uses and users. Hence, the activity focus provides a solution regarding the definition of the field (regime perimeter). This is especially useful in the case of artificial resources, where otherwise the list of all goods/services and users/owners of the resource is almost endless (e.g. collective housing). What is more, all the uses/goods and services might not even be very relevant for the research question under study.

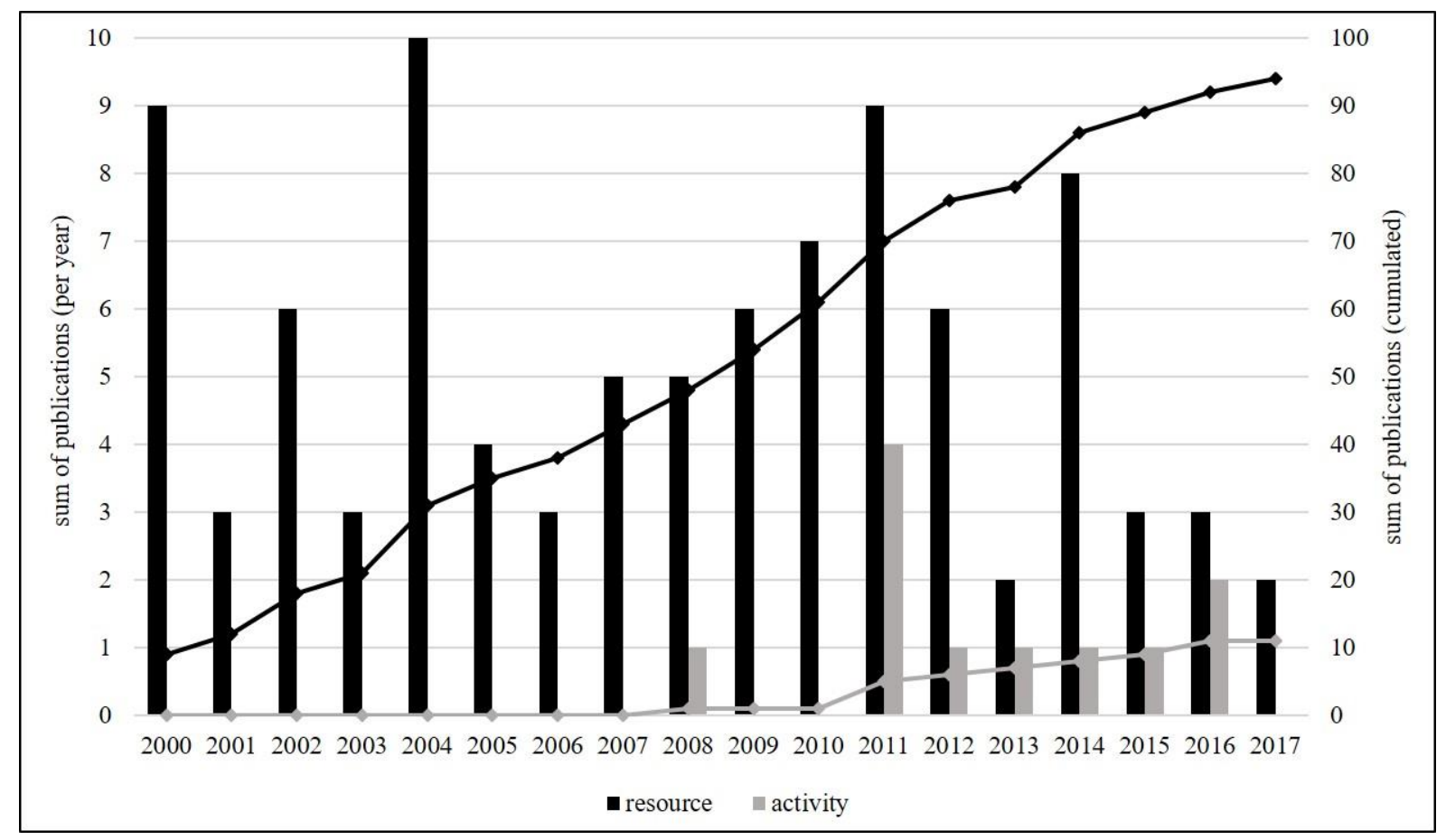

Figure 1: Focus of research: resource vs. activity $(n=108)$

Simultaneously with the "activity shift", we identify a change regarding the focus of the results in 2007 to shift toward actors. For the first time IRR research results focused on actors rather than resources. The number of publications focusing on both resources (e.g. the condition of the resource and stock) and actors (e.g. their strategies, conflicts), also increased over time (see figure 2). In line with the above findings on the shifts in focus on activity and actors, we also see that the relevance of actors has increased in IRR publications over time. The actors (users, owners etc.) play a role in the definition of an institutional regime, more specifically of the regime dimensions of coherence (degree of coordination between actors) and extent (number of goods and services regulated) (Gerber et al., 2009). From our review, we see that actors play a central role in the research design of over $70 \%$ of the articles. Despite this relative increase of relevance of actors in the research design, we see that overall, the results of publications still predominantly focus on resources (see figure 2). Specifically, we see a 
clear emphasis on resource conditions and less focus on conflicts or any other characteristics at the actor level.

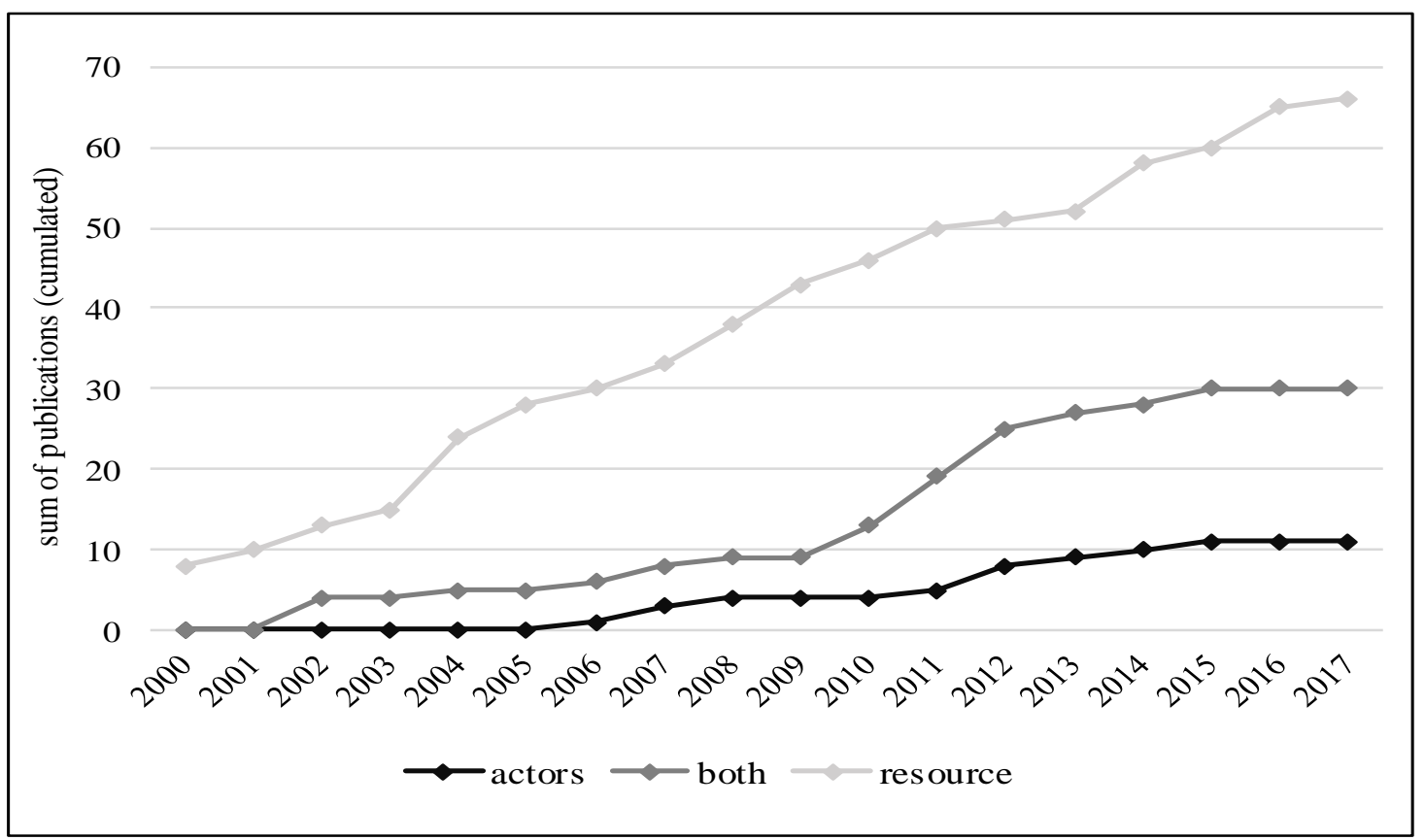

Figure 2: Relevance of actors in the results $(n=96)$

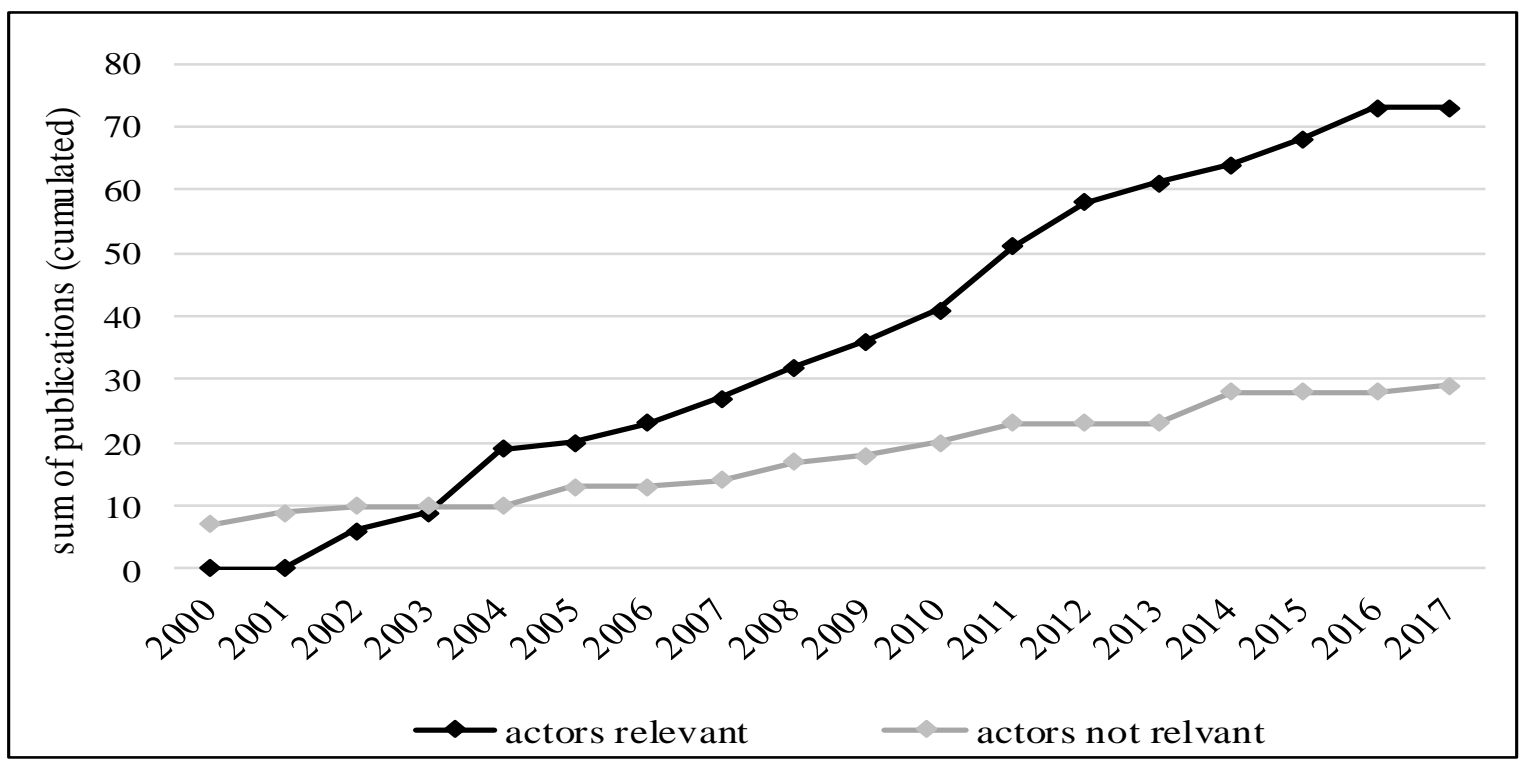

Figure 3: Relevance of actors in the research design $(n=102)$

In a similar vein, we also find that up until 2007 researchers mainly addressed rivalries within one resource. Then, around the same time as researchers began to focus on an activity as an entry point we find a tendency to also address issues between resources. An example is the study by Savary (2008) on the activity "mobility". The study addresses rivalry issues between 
the resource air and infrastructure (roads and land). An issue here is the definition of a resource. For example, a conflict over an artificial resource such as railway infrastructure, that includes multiple other resources (Moyson and Aubin, 2010), would then be a conflict "within" one resource, although multiple resources like land, forest and water are investigated.

The type of resource studied - natural or artificial resource - has also evolved over time (see figure 4). Results show that up until 2008, IRR scholars focused mainly on natural resources. Then we see a hiccupping trend to address artificial resources (such as housing or infrastructure projects), which peaked in 2011. However, researchers have been addressing artificial resources from the early days of IRR applications, although they did not always label them as such (Bisang et al., 2001, de Fossey, 2004). Despite these findings, the degree of applicability of the IRR for the study of artificial resources is contested (Knoepfel et al. 2001). Arguably, the IRR framework is interested in identifying all regulations specific to one resource (Knoepfel et al., 2001). Conversely, artificial resources contain multiple resources by definition (Nicol and Knoepfel, 2008). Again, an issue here is the inconsistency over time regarding what the researchers have defined as natural or artificial resources. For instance, researchers have defined landscape as a natural resource at the beginning (Knoepfel et al., 2001), but have later framed landscape as an artificial, secondary resource because cultural, aesthetical aspects were included (Rodewald, 2004).

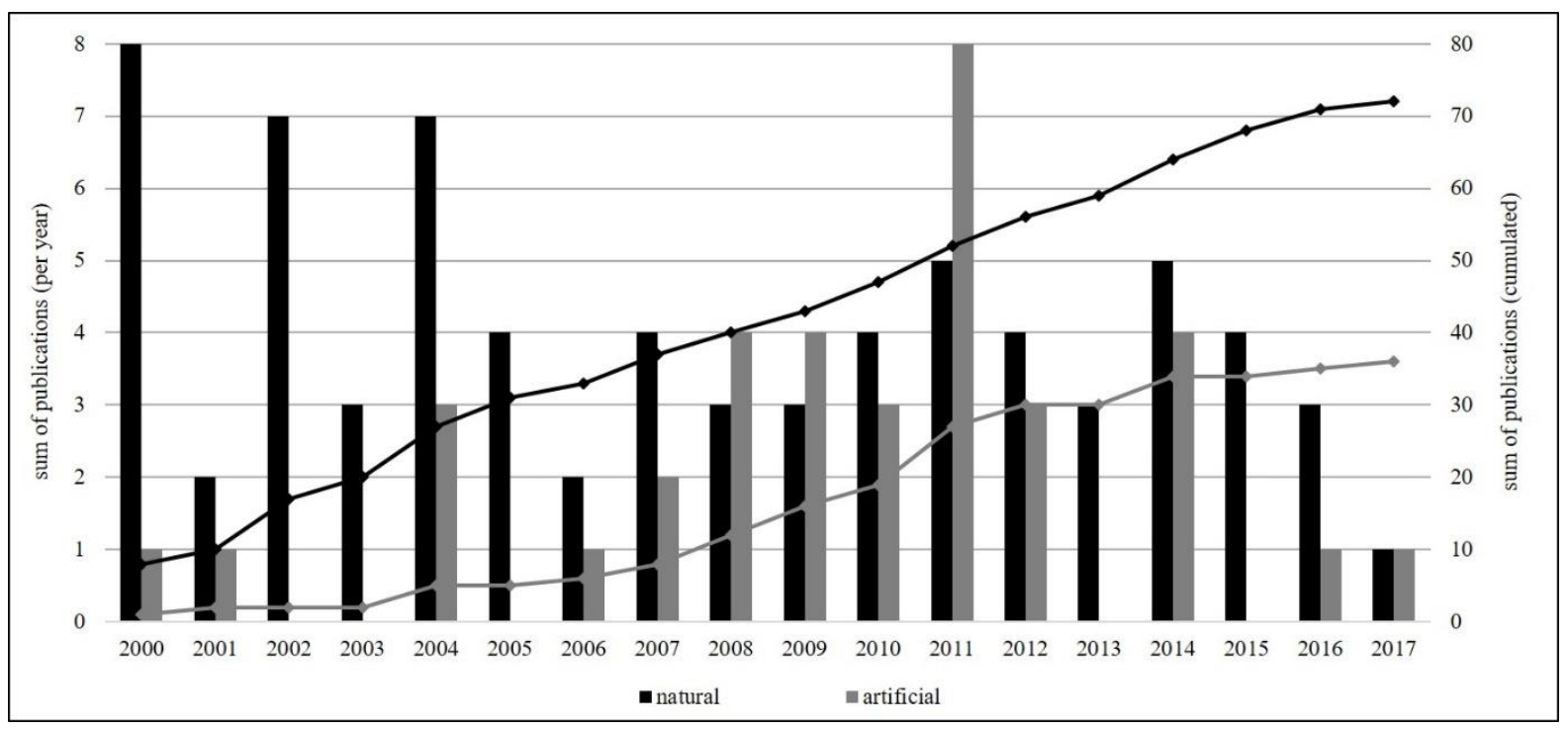

Figure 4: Type of resource: natural vs. artificial $(n=106)$

\subsection{Research approach and governmental levels}

We find that IRR scholars have taken a top-down approach (starting with a legal text) at the inception of IRR research and that this predominates over time. However, we recently observe 
an increase in bottom-up studies (starting with a resource and its use) as well as in mixed approaches, which take both a bottom-up and top-down perspective (see figure 5). However, the top-down approach also dominates publications where the local level is at the focus of research.

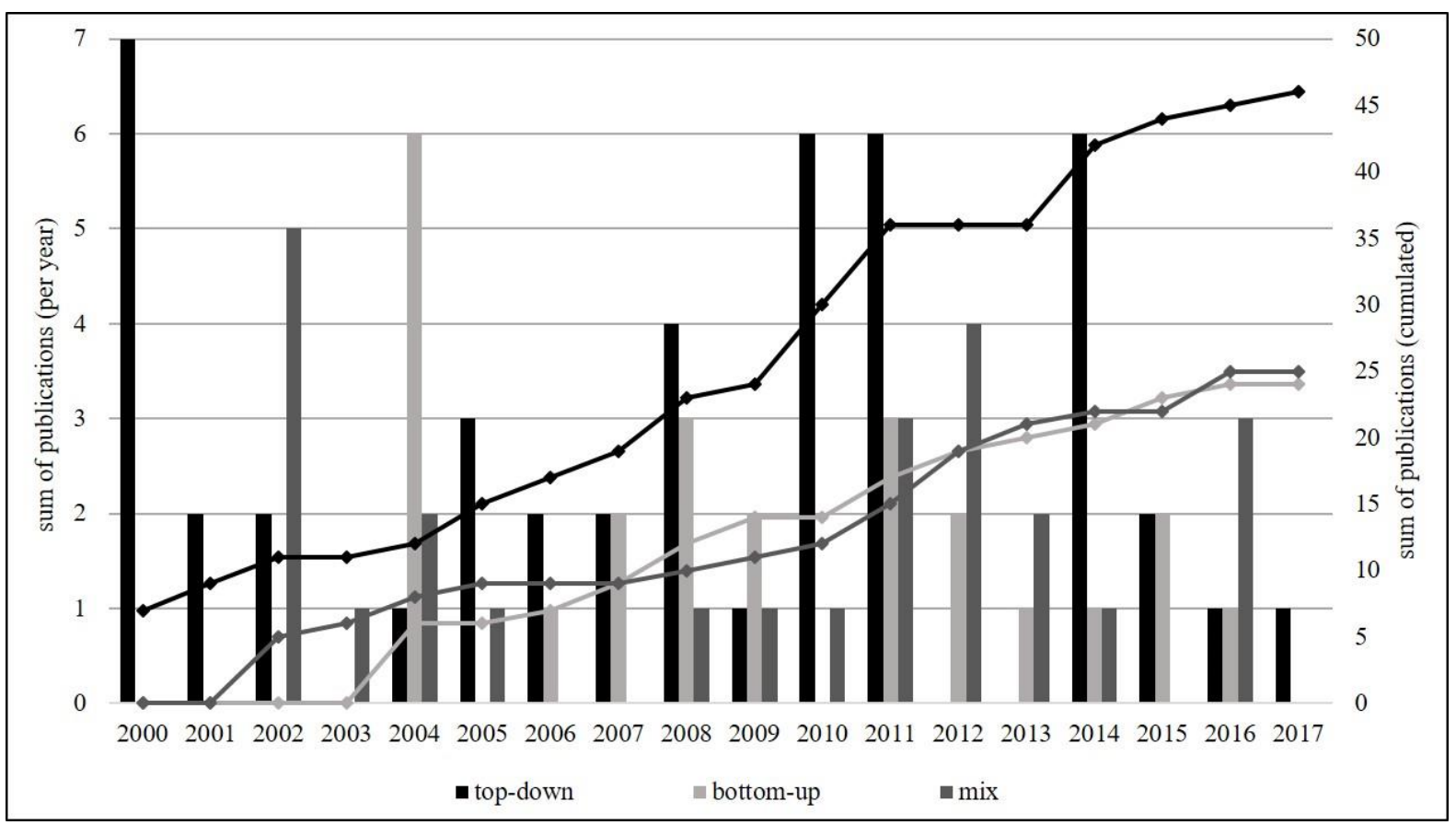

Figure 5: Research approach $(n=96)$

In terms of government level, figure 6 shows that IRR publications started with a national focus, but over time, the local level has become more important. Overall, we find local cases to predominate in our sample. This trend toward the local level again dovetails with the aspect of activity as an entry point, as the amount of local applications began to spike starting in 2007. With regard to the interplay between the levels, we find that about half $(51 \%)$ of the studies do not take an interplay into account while the other half does. 


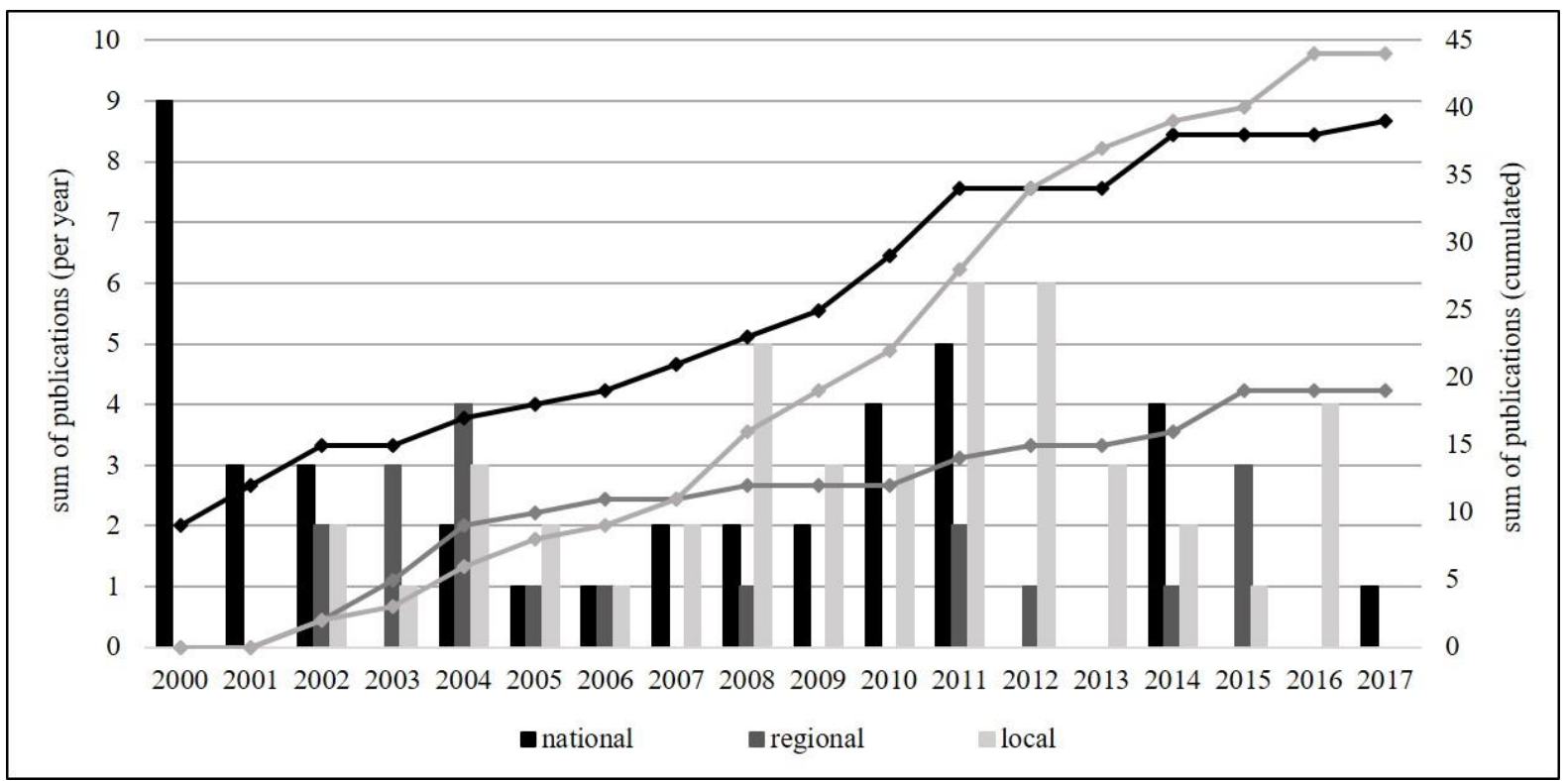

Figure 6: Government level of the case $(n=100)$

A final aspect of the research approach is that slightly more than half of the publications in our sample are comparative studies, while the other half of the analyses focuses on one case (see figure 7). The maximum number of cases compared is seven. We observe no change in this variable over time.

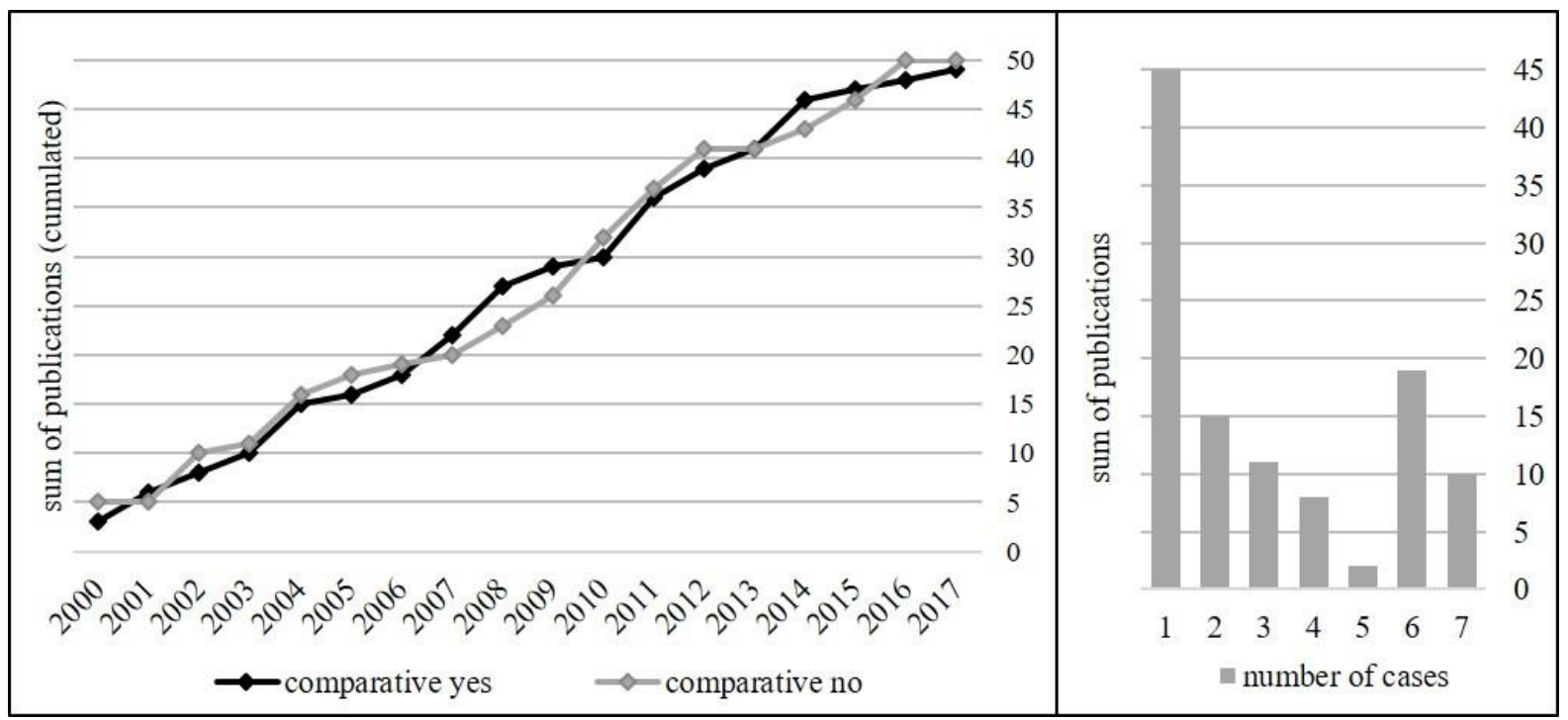

Figure 7: Comparative study yes or no $(n=99)$ and number of cases $(n=110)$ 


\subsection{Institutional regime, time and sustainability}

Regarding the time aspect in the research design, we find that the publications predominantly focus on the change of an IR (82\%). We find, however, that IRR scholars have defined change in a rather broad manner. It includes implementation at the local level (Aubin, 2007, Viallon, 2016, Garin and Barraqué, 2012), but also development of regimes over time (Kissling-Näf and Varone, 2000, Knoepfel et al., 2001, Kissling-Näf and Kuks, 2004). Scholars emphasizing activity over resources and applying a bottom-up approach typically focus on implementation at the local and regional levels. In contrast, scholars applying the top-down approach and focusing on resources, conducting the seminal historical screenings, focus on regime changes over decades (Kissling-Näf and Varone, 2000, Knoepfel et al., 2001). We then also find an interplay between different levels: Many publications focus on the question of how change (at a national or EU level) affects local resource management (Aubin, 2007, Viallon, 2016, Garin and Barraqué, 2012). We also find that slightly more publications employ the IR as the independent variable (41\%), than as dependent variable $(30 \%)$ and finally than both independent and dependent variable (29\%).

Almost all articles in our sample make a link to sustainability, even if it is just in the introduction and used as a "selling argument," which we coded as "mentioned" (see Figure 8). A minority (14\%) of publications do not mention sustainability. We can again link this with the start of scholars focusing on an activity and on actors: with an increasing focus on actors we find less focus on sustainability.

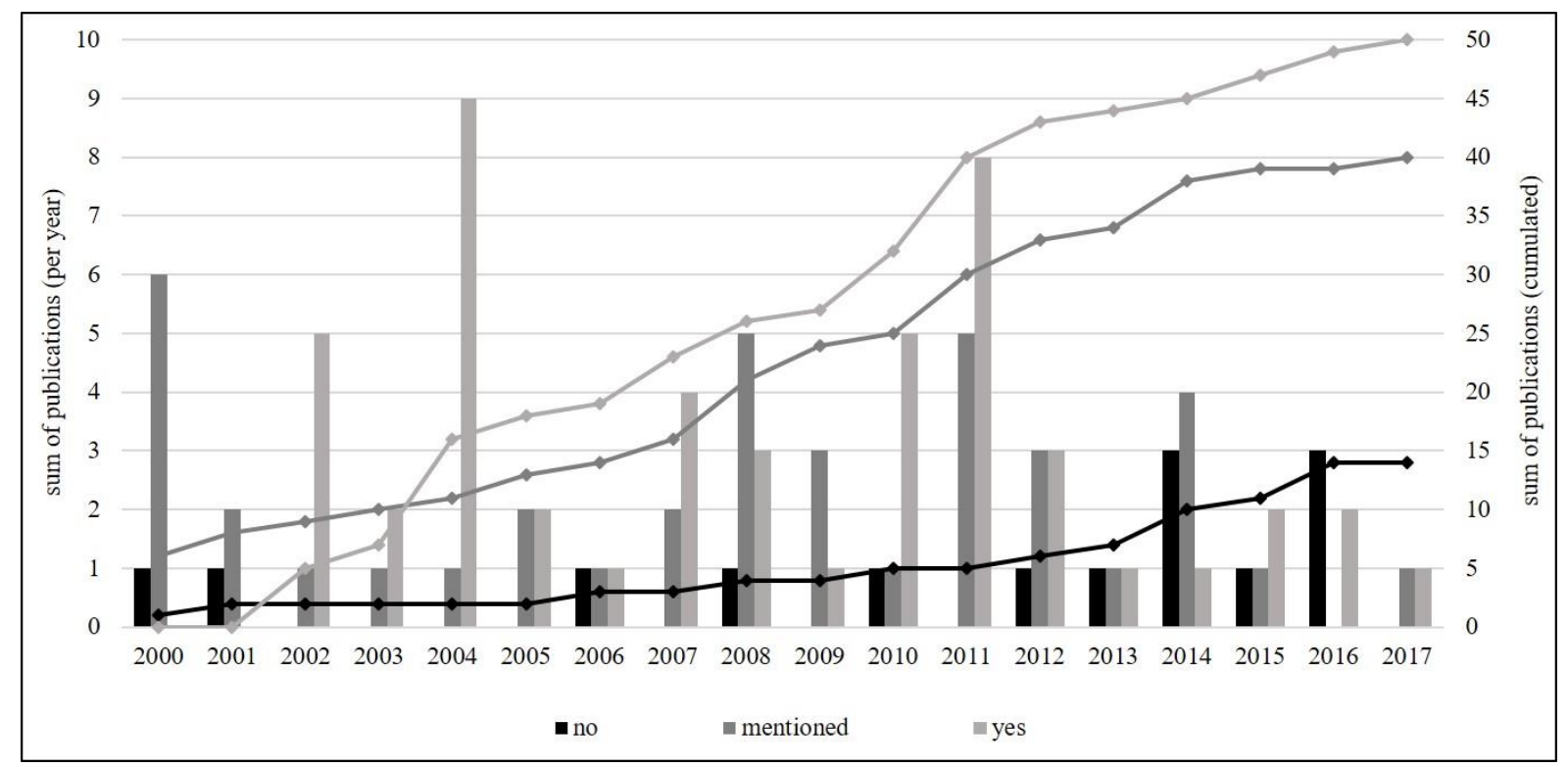

Figure 8: IR and sustainability $(n=104)$ 
Beyond making a link to sustainability, another intention of the IRR framework has been to show causality between the IR and sustainability. An example of an early study making this causal link is Bressers and Kuks' (2004) analysis of the impact of the adopted European Water Framework Directive on the sustainability of the resource water. As noted above, the causal link can go in two directions (following Gerber et al. 2009). A first option suggests that the closer the IR moves toward integration, the higher the probability that sustainable use conditions will be created (and vice versa). A second option hypothesizes that the greater the threat to the sustainability of the resource, the more likely it will be perceived as a collective problem, which again increases the probability of making the IR more sustainable (more integrated). We find that option 1 predominates, as only two articles have addressed option 2. Although many articles mention a causal relation between the IR and sustainability, only 35 discuss this aspect in their findings. We also find that sustainability is operationalized in different ways, such as indicator like air pollution (Savary, 2005), or broader indicators addressing the social, economic and ecological dimensions of sustainability (Schweizer and Knoepfel, 2011).

Overall, we find a trend of the IRR scholarship to focus more on activity and actors in the last decade, while the predominant focus of the publications across time (since the turn of the century) remains resource-centric. We surmise to link this increased focus on actors to the recognition that actors play a crucial role in resource management. Put differently, IRR scholars began to see actors as the missing link between laws and sustainability since around 2007 (de Buren, 2015). Since then, we see the development of two "camps" of IRR publications: those (older publications) focusing on historical analyses of resources based on legal texts and those (newer publications) addressing activities and putting actors more centre-stage. We can also link the research approach criterion to these two camps, whereby the former tends to take a top-down approach and the latter a bottom-up approach. We also see that most publications make a link to sustainability, although in recent years the trend to exclude sustainability has increased slightly.

\subsection{Structures of similarities and differences in the IRR literature}

For a more specific analysis of how different elements of existing IRR research relate to each other, we conduct on multiple correspondence analysis (MCA) (Greenacre and Blasius 2006, Le Roux and Rouanet 2010). MCA is a version of factor analysis designed for categorical variables. It allows detecting systematic patterns of similarities and differences between cases (IRR publications) in a statistically inductive manner and identifying elements (variables in the 
catalogue of IRR elements) that best describe these differences (see Trein et al., forthcoming)) and Exadaktylos and Radaelli (2009)) for applications of this method to literature review articles). MCA is based on a symmetric matrix of all two-way cross tabulations between the categorical variables. Based on this matrix, MCA identifies those categories that cluster together into a dimension (Trein et al., forthcoming). The analysis is performed with the FactoMiner and factorextra in R. As MCA is unable to deal with missing data, we relied on two steps to eliminate missing data from our dataset. First, cases with missing values on $50 \%$ or more of the variables ( 8 out of 110 cases) were excluded from the MCA. Second, the remaining missing data in our dataset was imputed with the missMDA package in $\mathrm{R}$. The procedure does not affect the dimensions of the MCA and results are based on the non-imputed data only.

The application of MCA to our dataset identifies two main dimensions (see Figure 9). The first dimension covers $20 \%$ of the overall variance between cases, the second dimension covers $12 \% .^{3}$ The subsequent discussion concentrates on these two dimensions, which together cover $32 \%$ of the overall variance between cases. Figure 8 shows the position of the variables - that is, of each category of the variables (see Appendix A for category codes) - in the twodimensional space created by the first two dimensions of the MCA. Colors represent the contribution of the variables to each dimension, with red colors indicating that the respective variable is important for the respective dimension. 


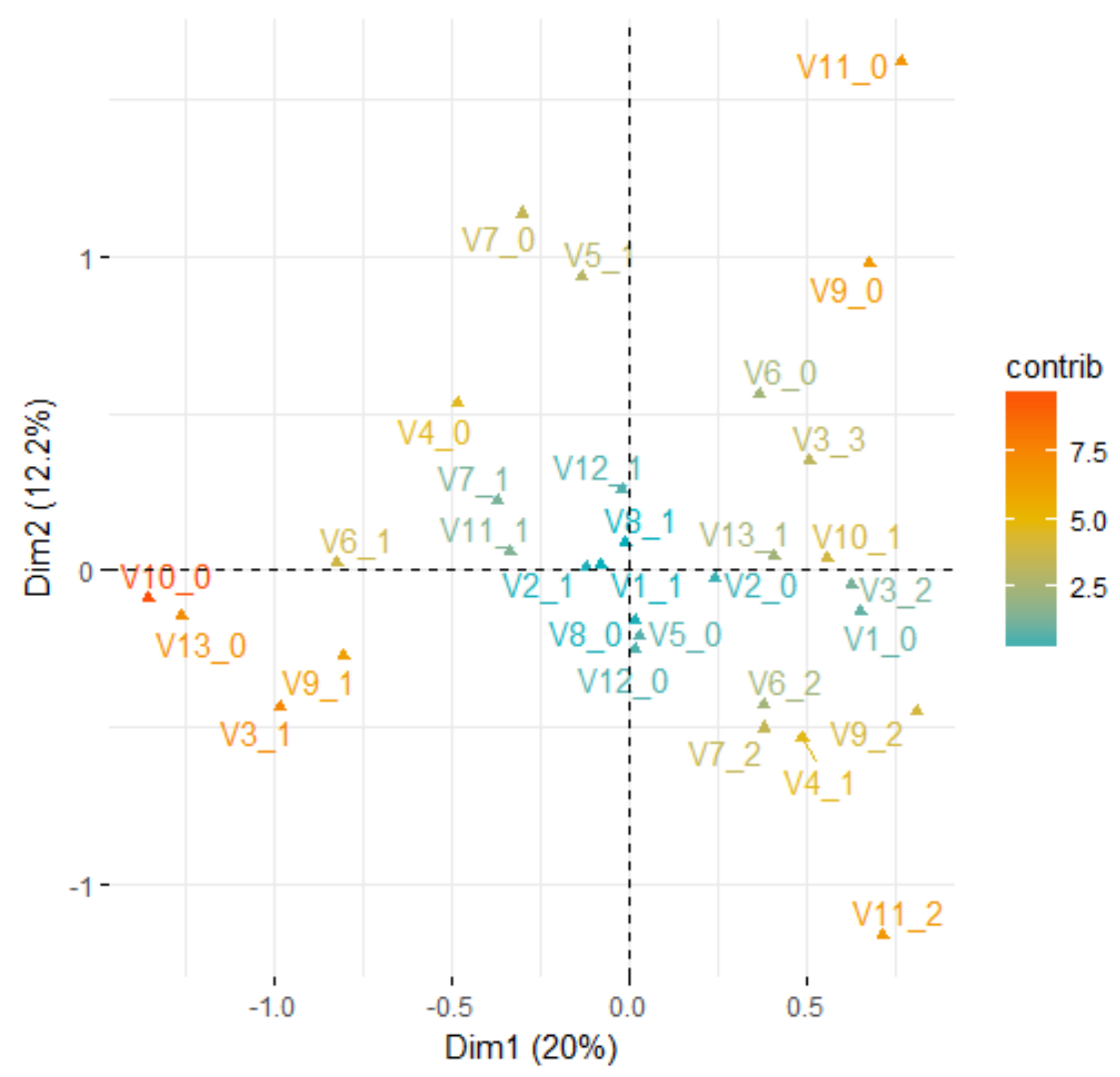

Fig 9: Variable categories on the two main dimensions

The clearly most important difference between studies that are on both extremes of the first dimension are a focus on actors (on top of figure 8) and a focus on both actors and resources (bottom of figure 8). Variables related to a focus on actors (V11_0) are studies where a bottom-up approach is chosen (V9_0), where sustainability is no issue (V7_0), and that focus on a stable IRR (V5_1) without studying level interactions (V4_0). By contrast, variables related to a focus on both actors and resources (V11_2) are those that define studies that take level interplay into account (V4_1) and that analyze sustainability issues (V7_2).

The second dimension then suggests - on the left side of the two-dimensional space a third group of studies wherein actors are non-relevant (V10_0), no analyses of actor relations as use rivalries or conflicts (V13_0), that focus on the national level (V3_1) and that rely on a top-down approach (V9_1). Other variables related to the left end of the second dimension are studies that use the IRR as the dependent variable (V6_1) and that do not analyze level interplay (V4_0). The right side of the second dimension is less clearly specified, as can be grasped from the absence of variables with a strong contribution (red) in figure 8 . The three corners of the distribution of variables and cases (not shown in figure 8) could be labelled as complex IRR studies (bottom right), top-down IRR studies (left) and bottom-up IRR studies (top right). The figure in Appendix B shows the distribution of the cases in the two-dimensional space. It shows 
that cases distribute roughly equally in the three camps, but also highlights that there are cases that do not clearly belong to any of the three camps (mostly the cases in the center of the figure, around the intersection of the two dimensions).

The following two cases provide an illustration of the group of complex IRR studies. Nahrath (2002) analyses the effect of the national regime change regarding spatial planning (between 1969 -1985) on the sustainability of the resource around and in Lausanne. Rivalries between the different uses of soil (e.g. urban use of rural soils) are addressed, the author follows a top-down approach and makes no statement regarding the causality or the direction of it between the regime (independent variable) and the sustainability of the resource (dependent variable). The focus of the results is on the resource not on actors. Nicol (2009) analyses the effect of changes in the regime of the artificial resource housing stock on a local level on actors' behavior in regard to the sustainability of the resource. Rivalries between actors (different uses) are addressed within the resource perimeter (e.g. type of construction and landscape, use of outdoor space for parking or playing etc.). Interplay between different government levels is no issue, the regime is both the dependent and the independent variable, sustainability is mentioned and the author follows a bottom-up approach and the focus of the results are on the resource, while actors play a role in the sense, that a regime change affects their behavior.

An illustrative example of the group of top-down IRR studies is the book by Knoepfel et al. (2001) which provides a historical analysis of the national regimes regarding the natural resources soil, water and forest. It follows a top-down, comparative approach, with the aim to identify the regimes (dependent variable) and triggers of change. Sustainability is mentioned, but not analyzed and actors are not in the focus. In the same time period Ammann (2000) provides a historical screening of the national regime regarding the natural resource air. Similarly to Knoepfel et al. (2001), the author takes a top-down approach, focusing on the resource, and the institutional regime is the dependent variable.

Finally, an illustrative case for the group of bottom-up IRR studies is the article by Garin and Barraqué (2012) on local implementation of the national regulatory framework regarding pollution of the resource water due to agriculture in France. The study assesses rivalries between users of different resources (land use and water) on a local level, includes the aspect of level interplay (including the international level through EU directives), follows a mixed approach and focuses on both the resource and actors (farmers and water service providers). Another exemplary case is the study by Bréthaut (2013) that analyzes how the national IR of water materializes locally in a touristic area. Rivalries between users and different uses (e.g. drinking water vs. hydroelectricity, agriculture vs. catchment protection) 
are taken into account. The effect of different types of local regulatory arrangements on the sustainability of the resource water is analyzed, following a comparative and mixed approach.

Overall, the combination of both dimensions and the suggested two-dimensional space with three corners nicely illustrates that the question of whether the focus of the research is on actors and activity or on resources, as well as related research design considerations of topdown and bottom-up approaches, define the key difference between the IRR studies analyzed in this article. This largely confirms our descriptive analysis above, albeit besides the two camps already indicated, it points to a potential third camp of complex IRR studies. Furthermore, it suggests certain trade-offs between elements that can be analyzed in IRR studies. Studies on the bottom-right appear as most complex: the focus of their results is on actors as well as resources, and they manage to take level interplay and sustainability issues into account. Both the group on the top-right as well as the group on the left clearly simplify the combination of the relevant IRR elements. Both do not consider level interactions and have a clear top-down or bottom-up starting point.

\section{Discussion and Conclusions}

We have taken stock of the existing IRR literature published since the turn of the century and draw lessons across a range of 110 publications. One important result is related to the focus of studies on actors and activities versus on resources. While the number of publications focusing on an activity as the entry point rather than resources remains small (10\%), we additionally find publications focusing solely on actors in their results (10.4\%) and another $14.5 \%$ focus on resources and actors (hence about 35\% of the sample in fact address activity and/or actors). Moreover, we find a trend to use activity as an entryway when applying the IRR framework since 2007. This dovetails nicely with other variables related to both the research design and findings such as the type of approach (bottom-up vs. top-down) and sustainability. For example, the pioneering IRR analyses focused on historical screening, taking a top-down approach by starting with the national legal texts. With the activity-focused research, we find a trend toward a bottom-up approach, starting from the resource used in the field and where institutions are activated by the actors, rather than first identifying existing institutions. Here we also find a tipping point in the manner that scholars have tried to explain sustainability, by including actors as part of the equation. Indeed, we find the means and possibility to explain sustainability, and causally link this to the IR, to be a contested issue in the IRR scholarship 
(de Buren, 2015). With our analysis, we cannot know whether this development in the IRR literature, that is, the increasing focus on actors and activities, corresponds to a spontaneous maturation of the field, or is rather the result of conscious decisions by leading researchers in the IRR community.

The analysis of the combinations of variables has suggested three groups of IRR publications that can be labelled complex IRR studies, bottom-up IRR studies, and top-down IRR studies. Potentially interesting combinations of variables that we do not observe, but would be interesting for future analyses include a focus on actors not only in the results but already putting a stronger focus on actors in the research design as well as taking an activity approach, while simultaneously addressing sustainability. This would be interesting because the studies focusing on actors and activity tend to argue that making a causal link with sustainability is a challenge (de Buren, 2015). Indeed, in our sample we see an increasing trend to exclude sustainability with a parallel trend to focus on actors and activity. It could thus be interesting for future studies to explain why this might be the case, by looking at examples of (un)sustainable resource management and looking for explanations at the actor level. Addressing this gap could also benefit from inputs by other frameworks such as Ostrom's work on polycentric governance (see Kellner et al., this issue). In addition, although actors are important in the IRR framework, none of the elements employed in this analysis provides insight regarding actors' beliefs, strategies and resources, which may be key in terms of the IR and (sustainable) natural resource management. Again, elements from other frameworks such as the Advocacy Coalition Framework (ACF, see Blake et al., this issue) could help to address such aspects. In addition, newer developments of the IRR such as work on Local Regulatory Arrangements also increasingly address aspects such as actor resources and strategies (see Viallon et al., this issue). 